\title{
Spatial location and its relevance for terminological inferences in bio-ontologies
}

\author{
Stefan Schulz*1,3, Kornél Markó1,2 and Udo Hahn²
}

\begin{abstract}
Address: ${ }^{1}$ Medical Informatics Department, Freiburg University Hospital, Freiburg, Germany, ${ }^{2}$ Language and Information Engineering (JULIE) Lab, Jena University, Germany and ${ }^{3}$ Master Program in Health Technology, Pontificial Catholic University of Paraná, Curtiba, Brazil

Email: Stefan Schulz* - stschulz@uni-freiburg.de; Kornél Markó - kornel.marko@uni-freiburg.de; Udo Hahn - Udo.Hahn@uni-jena.de

* Corresponding author
\end{abstract}

Published: 20 April 2007

BMC Bioinformatics 2007, 8:134 doi:10.1/86/1471-2105-8-134

This article is available from: http://www.biomedcentral.com/147I-2105/8/134

(C) 2007 Schulz et al; licensee BioMed Central Ltd.

This is an Open Access article distributed under the terms of the Creative Commons Attribution License (http://creativecommons.org/licenses/by/2.0), which permits unrestricted use, distribution, and reproduction in any medium, provided the original work is properly cited.
Received: 26 September 2006

Accepted: 20 April 2007

\begin{abstract}
Background: An adequate and expressive ontological representation of biological organisms and their parts requires formal reasoning mechanisms for their relations of physical aggregation and containment.

Results: We demonstrate that the proposed formalism allows to deal consistently with "role propagation along non-taxonomic hierarchies", a problem which had repeatedly been identified as an intricate reasoning problem in biomedical ontologies.

Conclusion: The proposed approach seems to be suitable for the redesign of compositional hierarchies in (bio)medical terminology systems which are embedded into the framework of the OBO (Open Biological Ontologies) Relation Ontology and are using knowledge representation languages developed by the Semantic Web community.
\end{abstract}

\section{Background}

Research activities in the "omics" sciences yield an evergrowing content of experimental data and publications, which are stored in a large variety of databases. For metadata descriptions and mediation between these resources a large number of bio-ontologies have evolved. In a similar vein, medical terminology and classification systems have been developed in order to improve medical documentation and data analysis.

Whereas naive methods of ontology engineering (typically, the ad-hoc assembly of concept trees and graphs) were prevailing in the past, the claim is increasingly expressed for a more principled approach, based upon logics and formal ontology design methodologies. (We here understand by formal ontologies representational artifacts which use a formal language to describe entities and their relations in the domain of choice.) The need for taming the mass of newly generated domain knowledge requires sophisticated and rigid computational methods to support semantic interoperability on a large scale. This can only be fulfilled when discretionary modeling decisions and implicit semantic assumptions underlying the assembly of terms and relations are avoided, as much as possible.

This paper is structured in the following way. First we will address taxonomies and partonomies as the main hierarchical principles in the context of concrete instances of bio-ontologies. We will introduce relations considered canonical to relate entities in our domain. In this context we will focus on boundary problems related to parthood and location. The central part of the paper is dedicated to the so-called "role propagation" phenomenon, a reason- 
ing pattern that is of high relevance for the domain. We will compare two approaches to represent this type of inferencing and provide recommendations for a parsimonious and ontologically adequate model.

\section{Taxonomic Aspects in Bio-Ontologies 2.I A Simple Upper Ontology}

In the following we focus on organisms and their physical components which are referred to as Biological Objects. According to a simplified upper ontology (see Fig. 1), which roughly follows the Basic Formal Ontology (BFO) $[1,17]$, Biological Objects are classified as Objects that are Independent Continuants. According to BFO, Continuants are those entities in the world that persist through time by being present in their entirety at every point in time at which they exist. Organisms, populations, organs, cells, cell components, molecules, and atoms are examples for BFO Objects. BFO Objects as Independent Continuants are opposed to Spatial regions on the one hand, and Dependent Continuants on the other hand. (Dependent Continuants are further divided into Qualities and Realizable Entities such as functions, roles, and dispositions, which are not an issue in this paper.)

At the uppermost level, we distinguish Continuants from Occurrents. According to BFO, Occurrents are those entities in the world which change in time. This means that each portion of the time during which an occurrent occurs corresponds to a temporal portion of the occurrent, because occurrents exist only in their successive temporal parts, phases [16], or stages [9].

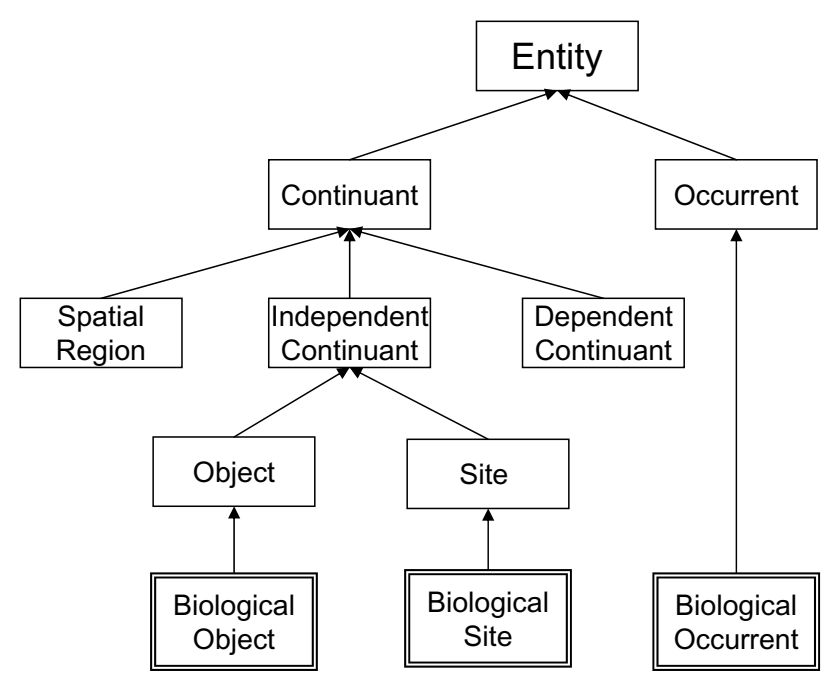

\section{Figure I}

A simplified upper ontology for biology. Siblings are disjoint but not exhaustive.
Occurrents are characterized by their ontological dependency on Continuants. For instance, every instance of the occurrent Cell Differentiation Process involves some instance of the continuant Cell.

As there is no uncontroversially accepted criterion for subdividing occurrents in terms of processes, events, changes, or actions, we do not make any further distinction.

Placing these categories into a context of biology, we end up with three disjoint (though not exhaustive) classes, viz. Biological Site, Biological Object, and Biological Occurrent, which are sufficient for our further considerations.

As familiar as the hierarchical arrangement of classes in a taxonomic framework may seem at the first sight, some clarification is necessary. First of all, the relationships between classes at the ontological layer and concrete individual entities in the world have to be characterized. We deliberately refrain from a principled discussion (which would have to cover nearly 2500 years of philosophy, beginning with Aristotle and addressing several controversial strands of philosophical thinking). We rather axiomatically introduce the relation instance_of, which relates an individual entity with the classes it belongs to. According to the OBO Relation Ontology [44], its domain and range do not overlap: No class can be instance_of anything, and individuals can never be instantiated. The division between classes and individuals is often treated in a fuzzy fashion, especially in naive approaches to ontology engineering. It may be tempting to describe, e.g. Serotonin_Receptor as instance_of Protein but then the representation of Serotonin_Receptor_2A would be impossible since an instance cannot be instantiated itself [33]. From a strict ontological point of view, even Serotonin_Receptor_2A would be a class (unless it is meant to refer to a single molecule or a defined amount of molecules, cf. [40]).

In line with the OBO Relation Ontology, we introduce the taxonomic subsumption relation $I s-A$ by the following axiom:

$$
\begin{gathered}
\forall A, B:\left(\operatorname{Class}(A) \wedge C \operatorname{Class}(B) \wedge I s \_A(A, B)=\right. \\
\text { def } \forall x:(\text { instance_of }(x, A) \Rightarrow \text { instance_of }(x, B)))
\end{gathered}
$$

This relation is equivalent to class subsumption in description logics [5] where the notation: A B is used.

Is-A relates specific with more general classes and it constitutes the organizational principle of taxonomic hierarchies. 
According to [44], classes and relationships between classes are represented by using italic font; we use bold face for all other relations.

\subsection{Common Bio-Ontologies and their Architecture} The class Biological Object (as introduced above) occupies a prominent space in bio-ontologies, covering the whole range from biomolecules to organisms. The pivotal role of Biological Object is due to the fact that its descendants form the backbone of any principled bio-ontological approach. Apart from the fact that the study of their structural properties is of utmost importance in molecular biology and genomics, biological functions inhere in biological objects, biological objects participate in biological occurrents, e.g. in biochemical pathways, and they are the subject to a broad range of experimental or therapeutic manipulations in biology and medicine.

There are numerous examples for Biological Object ontologies such as

- The Cellular Component branch of the Gene Ontology (GO) [14], describing species-independent relations between cell components;

- The ontology of Chemical Entities of Biological Interest (ChEBI) [12];

- Many animal and plant anatomies hosted by the Open Biological Ontologies (OBO) platform [23];

- The Foundational Model of Anatomy (FMA) [31,32], which describes the canonical anatomy of the adult human, with a focus on macroscopic anatomy;

- The "anatomy" schema of the GALEN CORE model $[2,29]$;

- The "anatomy" part of the NCI Thesaurus [18];

- The "anatomy" branch of SNOMED CT [3].

As a kind of unifying principle, the nodes in ontologies of biological objects are mostly arranged in a directed acyclic graph, using a bipartite hierarchical structure:

Taxonomic links associate specific with general classes using the relation $I s-A$, whereas mereological links [46] associate parts and wholes.

\section{Mereological Aspects in Bio-Ontologies 3.I The Meaning of "Part"}

According to our stipulations, relations between parts and wholes require a principled account under the following aspects:
1. There must be a clear commitment to formal properties, i.e. in terms of transitivity, reflexivity and symmetry, as well as domain and range.

2. The wide-spread use of relations such as "part-of" and "has-part" between classes seems intuitive at a first sight, but is ambiguous and contrasts with the classical approach to mereology [43], which focuses on individual entities, and not classes of entities.

3. It has to be made explicit whether parts and wholes should be understood in a either functional or a purely locative sense and how temporal aspects are treated. The boundary to other relations should be made clear.

None of the ontologies of biological continuants mentioned above make sufficient claims regarding these three controversial issues.

FMA and GO have a clear commitment to item 1, at least regarding the transitivity property of the general "part-of" relation. The same applies to GALEN with regard to "partof" subrelations [29]. This view is mainly consistent with classical (i.e., axiomatic) mereology $[10,43]$ which treats generic parthood as reflexive, antisymmetric and transitive, with proper parthood as its irreflexive variant, excluding equality. It is the latter one which better fits the habitual understanding of "part-of" in biology. In contrast to this, the OBO Relation Ontology has recently introduced the "part-of" relation as a reflexive one [44].

Concerning other formal properties, such as extensionality, supplementation, or the treatment of time $[7,9,43]$, none of the specialized biomedical terminology system takes up a defined position. With regard to the time aspect, the OBO Relation Ontology introduces the relation derives-from which includes the notion of what can be called historic parthood. Hence a DNA extracted from a cell is no longer "part-of" it. Implicitly, the mereological relations are restricted to continuants. Parts of occurrents are considered in SNOMED CT [3] but they are not identified as such [39].

Relating classes using mereological relations (item 2) is very common. However, semantic network style assertions such as part-of(CellNucleus, Cell), are so ambiguous that conflicting interpretations are likely to evolve: So did the Gene Ontology use to define this relation as follows: "part-of means can be a part of, not is always a part of". In the meanwhile this definition has changed to "The part-of relationship used in GO is usually (...) necessarily is part" [15]. In contrast, the Foundational Model of Anatomy (FMA) [32] uses class-level mereological relations in the following way: part-of $(A, B)$ means that any instance of $B$ has an instance of $A$ as part and any instance of $A$ is part 
of an instance of $B$ [45] (This leads to problematic assertions such as part-of(Rectum, Female Pelvis)).

In this paper, we exclusively use mereological relations which hold between individual entities. Wherever they are used in class definitions quantifications will be used.

A rudimentary commitment to item 3 is reflected by the introduction of several mereological subrelations in FMA and GALEN [30]. For a more recent discussion of such subrelations, with a strong emphasis on biology, we refer to $[8,21,26]$ who focus on the transitivity of mereological relations. According to [47], apparently plausible statements such as "The nucleus of a cell is not a part of the organ which that cell is part of " do not really challenge the assumption that the classical parthood relation is truly governed by formal ontological principles. It is rather the expression of an implicit narrowing of the sense of "part", which commonly happens in ordinary discourse. Taking the above statement, it holds true if we read "part" as "functional part", but not if we consider parthood in the broadest sense.

We here follow the OBO recommendations [44] and axiomatically introduce the relation part_of and its inverse has_part as transitive, antisymmetric, and reflexive relations which can be asserted either between pairs of continuants or between pairs of occurrents.

In addition we introduce the irreflexive parthood relations proper_part_of and has_proper_part. Parthood relations between classes are not considered because they can be defined in terms of part_of such as introduced in [44].

\subsection{The Puzzle of the Parthood/Containment Distinction in Biology}

When we describe biological objects, it is often difficult to decide whether two objects are related in terms of parthood or in terms of a weaker relation of spatial inclusion. To a much higher degree than can be observed with artifacts, living biological entities continuously exchange matter with their environment. Typical examples are phagocytosis, pinocytosis, and exocytosis, such as depicted Fig. 2 and Fig. 3. In contrast to artifacts, there is
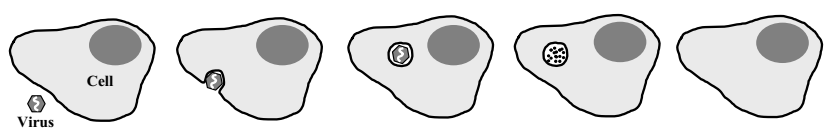

Figure 2

Phagocytosis of a virus by a cell: At which stage(s) one can state that components of the ingested virus are not only contained in but also part_of the cell?

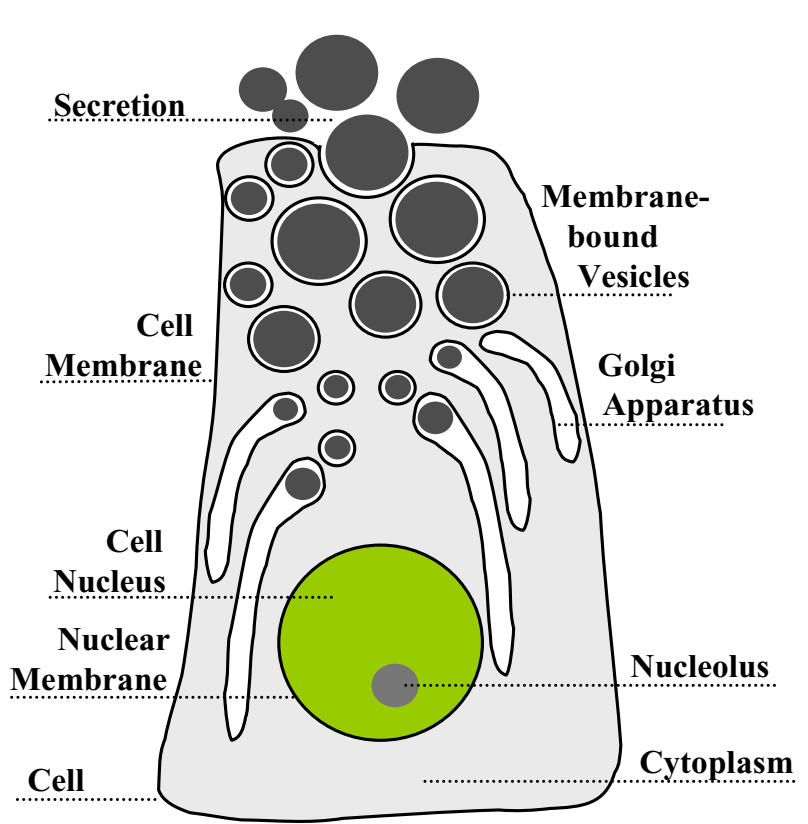

\section{Figure 3}

Prototypical Cell from a salivary gland: Are the secretions produced in the cell also part_of the cell or are they merely contained within them?

no clear distinction between a system's constituents and its substrates. In contrast, as far as a mechanical engine is concerned, the material of which it consists is clearly distinct from the fuel it consumes and the exhaust-gas it produces.

Let us consider the following example: A carbon atom (e.g. as part of a carbohydrate) is ingested through the alimentary system where it can play quite different roles: It may merely participate in the organism's "power supply", after which it is eliminated by the lungs as part of a $\mathrm{CO}_{2}$ molecule or it may be integrated into the organism's constitutional structure, e.g. as part of a collagen fiber which then remains stable in the organism for years (cf. [35] for more examples of this kind). Other controversial examples on the cell level include small molecules and ions, as well as endosymbionts like mitochondria and chloroplasts. Another example is the reproduction process: A spermatozoon fuses with an oocyte then the male and female pronuclei form and merge. Afterwards the chromosomes are re-arranged, a zygote is formed and, finally, its cleavage is initiated. So it can be controversially discussed at which phase of this process which components of the original oocyte cease to be part of the female organism in which the oocyte has developed.

Organisms interact with other organisms as well. If a virus is in a cell, it is certainly not part of this cell. At which stage 
of the process of digestion (phagocytosis) do virus or bacteria components become parts of the ingesting cell (cf. Fig. 2) is difficult to delimit. Considering the cells which produce secretions (cf. Fig. 3), are these substances part of those cells or are they merely located within them? Many tissues such as the intestinal mucosa or the endometrium undergo permanent renovation, i.e. discharge of cells. Are these cells still part of the original tissue or not?

\subsection{Spatial Location}

In order to bring light into the parthood/containment dilemma, we introduce location as a new primitive for spatial aggregation of biological objects. Every instance of a continuant or an occurrent occupies a unique spatial region at every moment in time at which it exists. According to the Basic Inclusion Theory (BIT) [11] we introduce here the primitive region_of $(e)$, a function of an individual entity $e$, the value of which is the spatial region $s$ that $e$ fully occupies. This corresponds to the region function $r$ in BIT. In contrast to BIT, we here also allow occurrents to have a spatial location. We here define the region an occurrent fully occupies as the mereological fusion of all regions occupied by any stage (instantaneous part [9]) of this occurrent during its lifetime.

Using this notion of region, we define the relation located_in between two entities as follows: entity $x$ is located_in entity $y$ if and only if the region occupied by $x$ is part of the region occupied by $y$.

$$
\begin{gathered}
\forall x, y: \text { located_in }(x, y)= \\
\text { def part_of(region_of }(x), \text { region_of }(y))
\end{gathered}
$$

According to this, the located_in relation holds between any two entities that occupy the same region. We do not discuss here whether it makes sense - according to our definitions - to talk about the location of an occurrent in another occurrent, or about an continuant in an occurrent. The only case we consider here concerns the location of a continuant in another one, or the location of an occurrent in a continuant. Location can also be asserted between regions since, according to BIT,

$$
\forall x \text { : region_of(region_of }(x))=\text { region_of }(x)
$$

We complete the picture by adding location_of, the inverse relation of located_in. Since the region occupied by a part of an object is always part_of the region occupied by the including whole, it follows that the relation part_of is a specialization of the relation located_in:

$$
\begin{gathered}
\forall x, y: \text { part_of }(x, y) \Rightarrow \text { located_in }(x, y) \\
\forall x, y: \text { has_part }(x, y) \Rightarrow \text { location_of }(x, y)
\end{gathered}
$$

The relation located_in is further subdivided into part_of and contained_in. If an object is contained_in another one it is located_in it without being part of it. A distinction between part_of and contained_in is only possible for location between Objects, cf. [41].

$$
\begin{gathered}
\forall x, y: \text { contained_in }(x, y)==_{\text {def }} \\
\text { instance_of }(x, \text { Object }) \wedge \text { instance_of }(y, \text { Object }) \\
\wedge \text { located_in }(x, y) \wedge \neg \text { part_of }(x, y)
\end{gathered}
$$

It still remains difficult to distinguish parthood from location in an objective manner. In [41] we proposed a set of criteria which help decide whether a location relation between two objects $a$ and $b$ can be refined to a parthood relation. One criterion is the consideration of the objects' lifecycles. If $a$ is always located_in $b$, then it can be regarded as part_of $b$. Another criterion we proposed is functionality. If $a$ is located_in $b$ and $a$ functionally contributes to $b$ then $a$ is part_of $b$, at least if the function is affected when $a$ is missing. Especially the latter criterion depends on an ontologically stable approach to biological function which is still due.

Finally, we introduce the primitive $\mathrm{OBO}$ relation pair participant_of and has_participant. This relation is asserted whenever biological objects are "participants" of an occurrent Unfortunately, OBO only vaguely characterizes this relation, namely "a primitive instance-level relation between a process, a continuant, and a time at which the conobtains, for example, when this particular process of oxygen exchange across this particular alveolar membrane has_participant this particular sample of hemoglobin at this particular time" [34]. We here understand the participation relation as a common subsumer of the 'agent' and 'patient' relations, as known from verb semantics [13].

Here, the agent is the instigator of an action, and the patient is the entity that undergoes any impact of an action or an event. We consider the relation has_participant in the context of biomedicine to relate a biological occurrent to those objects that are directly involved in it. For instance, a phosphorylation event has a kinase, a $\mathrm{PO}_{4}$ group, and an organic molecule as participants. Direct involvement has an important corrollary: The extension of participating objects is limited to the region where the occurrent is located. Therefore, if a single membrane protein is phosphorylated, neither the whole membrane nor the whole cell are considered participants of this phosphorylation event. In a similar vein, if a toe is amputated, the toe itself but not the whole foot acts as a participant. 


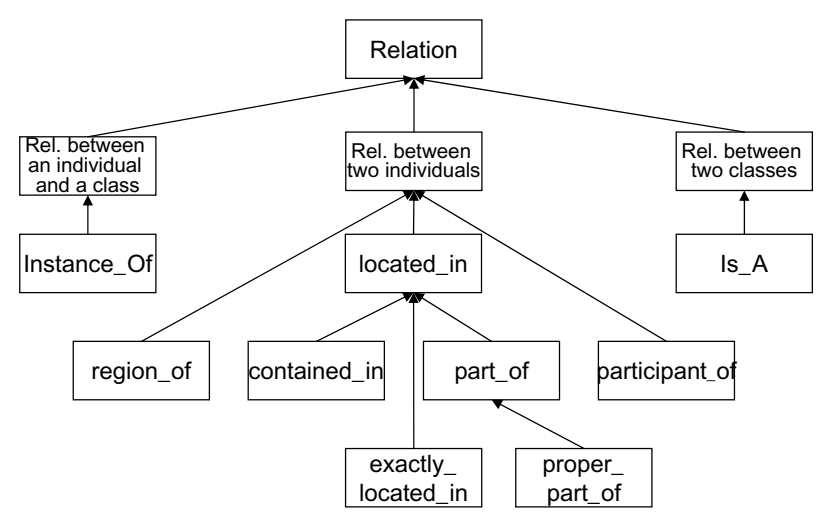

Figure 4

A simplified Relation Ontology for biology. Sibling relations are disjoint but not exhaustive.

Fig. 4 depicts the taxonomy of the relations which we use throughout this paper. Note that we have restricted ourselves to binary relations and that we ignore - for the sake of simplicity - the dimension of time. The relations follow the OBO suggestions, as introduced by [44], as well as the BIT, suggested by [11].

\subsection{Patterns of Taxonomic and Partonomic Reasoning}

We now address ourselves to the main topic of this paper, $v i z$. the discussion how taxonomic and partonomic reasoning patterns interact and how plausible inferences can be drawn.

While reasoning in taxonomic hierarchies is relatively well understood - each instance of a specific class is also an instance of any more general class, and thus inherits all of its properties [24] - we lack an equal form of consensus for part/whole-related reasoning (cf. [4] for a survey).

The embedding of part/whole-related reasoning into more general mereological reasoning, i.e., the relation between parts and wholes and the space they occupy, are difficult issues in the construction of formal ontologies $[10,11,38,41]$. This is especially relevant when it comes to the representation of biological occurrents and their dependence on biological objects.

A frequently mentioned reasoning pattern is the so-called role propagation along mereological hierarchies. The importance of this problem is well accepted. It has led to the introduction of dedicated constructors in early knowledge representation languages, such as transfersThru in CycL [22] and specializedBy in GRAIL [28]. In a nutshell, role propagation is a reasoning phenomenon that is best explained by the following prototypical example:
"Every instance of 'fracture of the neck of the femur' is also considered an instance of 'fracture of the femur' because every neck of a femur is a part of some femur." [19]

Of course, this pattern is not restricted to reasoning about medical disorders and procedures but it extends to biology as well. So the biological occurrent of Gastrin Secretion is considered a process located at Gastric G Cells of the Gastric Mucosa, and therefore, we naturally classify it as a process located at some Gastric Mucosa since every instance of Gastric G Cells is part of some Gastric Mucosa.

In our previous research on biomedical knowledge representation [36-38,41] we have already addressed this issue extensively. We here provide a coherent account of modeling parthood in biological continuants in terms of spatial location. Based on the discussion of the nature of partwhole relations for biological continuants, we stipulate that for the purpose to support these inference patterns, the relations between wholes and their associated parts can be abstracted in terms of spatial relations, so that subsumption-based reasoning patterns can be reused to propagate roles across mereological hierarchies. The above reasoning pattern, which can be paraphrased as " $x$, which is related to $y$, is related to $z$ as well because $y$ is a part of $z$ ", is equivalent to the so-called right identity rule [6]:

$$
\forall x, y, z: \operatorname{rel}(x, y) \wedge \text { part_of }(y, z) \Rightarrow \operatorname{rel}(x, z)
$$

The symbol rel stands for an arbitrary relation. This is instantiated by the following examples.

- "Glomerulonephritis is a kind of nephritis because glomerula are parts of kidneys and nephritis is an inflammatory disorder of the kidney."

- "Insulin production is usually considered a pancreas process, because pancreatic beta cells are located in the pancreas".

However, there are counterexamples:

- "An amputation of a toe is never a foot amputation, although every toe is part of a foot."

- "Mitosis is a cell process but it is generally not seen as a pancreas or liver process although these organs have cells as parts."

- "DNA replication cannot be not subsumed by cell replication although DNA is located in a cell."

This kind of anomaly make the usefulness of right-identity rules problematic [27], since there is ample evidence 
that such role inclusions do not always hold [38], as our examples suggest.

Let us analyze another example in a more principled way: Every instance of the class Gastroenteritis is an instance of Inflammatory Disorder related to some instance of Digestive Tract as a whole, and therefore, it is not identical with any instance of the class Appendicitis, which is defined as an Inflammatory Disorder of the Appendix - although every instance of Appendix is part of some instance of Digestive Tract. On the other hand, every instance of Nephritis is an instance of Inflammatory Disorder of some instance of either some Kidney or any of its parts. As a consequence, the class Nephritis subsumes Glomerulonephritis because every instance of Glomerulonephritis is an Inflammatory Disorder of some instance of Glomerula, each of which is part of some Kidney.

Obviously, some definitions relate to some entity type as a whole, whereas other ones relate to the whole or any parts of it.

In our previous work $[37,38]$ we preferred to separately address the class of "a whole" (the so-called E-node), the class of "the proper parts of a whole" (the so-called Pnode), and the class of "the proper or improper parts of a whole" (the so-called S-node).

We argued that the introduction of artificial classes (which are defined as reifications of roles) is epistemologically valid, thus justifying the so-called SEP-triplets [42] approach. Fig. 5 (upper part) depicts a SEP-triplet model. Occurrents are linked to objects by a very general and not further defined relation associated_with. The burden of reasoning lies on the taxonomic structure. Since Nephritis is connected to the S-node class Kidney Structure and Glomerulonephritis is connected to the S-node class Glomerulum Structure which is subsumed by Kidney Structure, one can infer that Glomerulonephritis is a subclass of Nephritis. This reasoning pattern is not supported for Nephrectomy because here the target is Kidney as a whole and not a proper part of it. This approach was proposed in order to overcome the lack of former terminological languages which did not allow to directly specify algebraic properties of relations, such as transitivity or reflexivity. It has been taken up in the meantime by clinical terminologies, e.g. SNOMED CT [3].

However, the SEP approach is controversial mostly due to several facts. Firstly, it requires extensive modification of the class hierarchies. Secondly, the semantics of the extra nodes (S-Nodes and P-nodes) is frequently misunderstood. (In SNOMED CT, the same medical terms are assigned to E-nodes and S-nodes.) Finally, one can raise objections from a standpoint of formal ontology: The SEP

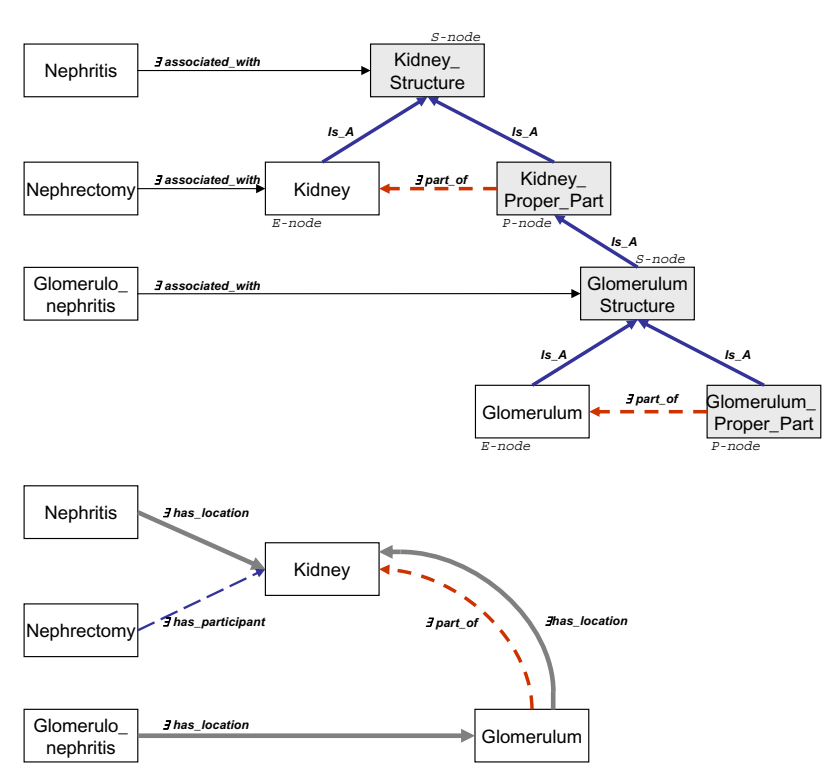

Figure 5

Top: SEP triplet approach for role propagation. Bottom: Alternative solution with semantically clear-cut relations. The relations labels use description logics [5] notation.

model addresses (and solves) certain reasoning requirements in a superficial way, i.e. it does not require a formal analysis of the relations involved.

Furthermore, there are now terminological languages such as $\mathcal{E} \mathcal{L}$, implemented in efficient reasoners, e.g. CEL [6] which support right-identity rules.

In the following, we propose an alternative solution to this problem where we completely refrain from complicated modeling artifacts. We show instead how the required inferences naturally derive from precise class definitions, using a parsimonious set of relations with welldefined algebraic properties. We further enhance our picture by extending the approach to disposition properties and their propagation along inclusion hierarchies.

\section{A Formal Approach to Role Propagation across Partonomies}

4.I Integrating Taxonomic and Mereological Hierarchies

As we have shown in Section 3.1, the compositional structure of classes of biological objects cannot sufficiently be described by one single mereological hierarchy. As much as we can derive that an individual $b$ has an individual $a$ as part, knowing that $a$ is part of $b$, we cannot transfer the same reasoning pattern to the level of classes.

Here, if we relate classes by quantified relations we can not infer that every instance of $B$ has always some instance of $A$ as part, knowing that every $A$ is part of some $B$. (Using 
DL notation [5], $B \quad \exists$ has_part. $A$ does not imply $A$ $\exists$ part_of.B.)

However, to the moment, the recommendation to treat links from the part to the whole differently from links that point from the whole to the part - although principally allowed at least according to the Gene Ontology usage guidelines [15]- has not been taken up by any of the OBO ontologies. Fig. 6 demonstrates how this might look like, using a couple of Gene Ontology classes. The partonomic relations between Nuclear Membrane and Cell Nucleus are bidirectional: every Nuclear Membrane is part of some Cell Nucleus, and every Cell Nucleus has some Nuclear Membrane. This is not the case with the classes Cell, Membrane, and Cell Projection. Not every Cell has a Cell Nucleus or a Flagellum, Axon, or other Cell Projection, but all of them are part of some Cell. Every Cell has some Membrane, but not every Membrane is part of some Cell.

These subtle though important partonomic distinctions have to be accounted for when we want to support plausible inferences involving biological objects and biological occurrents.

When we refer to the location of biological occurrents at a certain part of an organism or a cell we mean, more precisely, the location of this part. For this relation, we use the predicates location_of and located_in, in their broadest sense, including biological occurrents or dispositions: A biological object can therefore be the location of some biological occurrent, e.g. every instance of a biological occurrent $P$ (e.g. rRNA transcription process) is located at some physical location E (e.g. RNA Polymerase I).

$$
\begin{aligned}
\forall x: \text { instance_of }(x, P) \Rightarrow & \exists y: \text { instance_of }(y, E) \\
& \wedge \text { located_in }(x, y)
\end{aligned}
$$

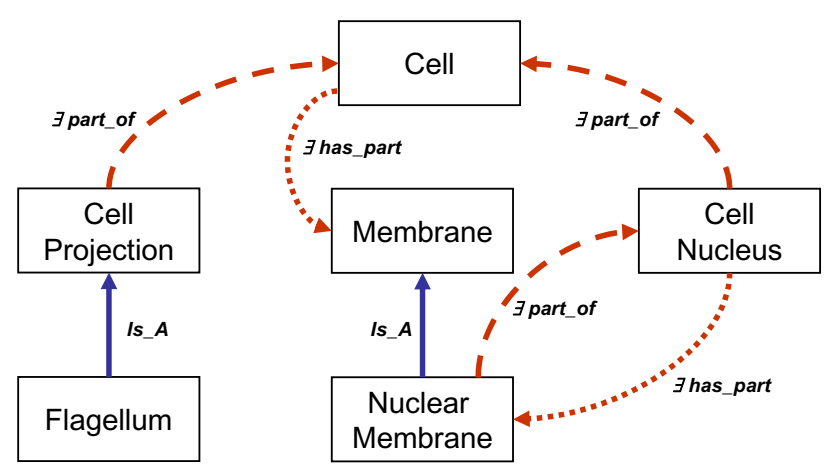

\section{Figure 6}

A complete mixed taxonomy/partonomy. In contrast to the OBO ontologies, Part_Of hierarchies here do not always coincide with Has_Part hierarchies. The relations labels use description logics [5] notation.

\subsection{Inferences involving Biological Occurrents}

We now provide examples for plausible inferences, commonly referred to as propagation of properties.

\section{Example I}

"A nephritis is an inflammatory disorder located at a kidney as a whole or at any of those objects which are necessarily part of a kidney."

$$
\begin{gathered}
\forall x: \text { instance_of }(x, \text { Nephritis }) \Leftrightarrow \\
\text { instance_of }(x, \text { InflammatoryDisorder }) \wedge \exists y: \\
\text { instance_of }(y, \text { Kidney }) \wedge \text { located_in }(x, y)
\end{gathered}
$$

The inference that every Glomerulonephritis is a Nephritis is then straightforward. The statement

$$
\begin{gathered}
\forall x: \text { instance_of }(x, \text { Glomerulum }) \Rightarrow \\
\exists y: \text { instance_of }(y, K i d n e y) \wedge \text { located_in }(x, y)
\end{gathered}
$$

can be inferred from

$$
\begin{gathered}
\forall x: \text { instance_of }(x, \text { Glomerulum }) \Rightarrow \\
\exists y: \text { instance_of }(y, K i d n e y) \wedge \text { part_of }(x, y)
\end{gathered}
$$

because part_of $(x, y) \Rightarrow$ located_in $(x, y)$. Glomerulonephritis is defined as:

$$
\begin{gathered}
\forall x \text { : instance_of }(x, \text { Glomerulonephritis }) \Leftrightarrow \\
\text { instance_of }(x, \text { InflammatoryDisorder }) \wedge \\
\exists y: \text { instance_of }(y, \text { Glomerulum }) \wedge \text { located_in }(x, y)
\end{gathered}
$$

Due to the transitivity of the relation located_in and the fact that every instance of Glomerulum is located in some Kidney (Formula 10), we get:

$$
\begin{gathered}
\forall x: \text { instance_of }(x, \text { Glomerulonephritis }) \Rightarrow \\
\text { instance_of }(x, \text { Nephritis })
\end{gathered}
$$

The relation located_in is used both for the spatial location of Glomerulum in Kidney and for the relation between the occurrent Glomerulonephritis and Glomerulum. So it is uniquely the transitivity property of the relation located_in which provides the required inference.

\section{Example 2}

"Apoptosis is a kind of cell death. Tissue has cells as parts. Even though, apoptosis is not a necrosis (tissue death)".

$$
\begin{gathered}
\forall v \text { : instance_of }(v, \text { Necrosis }) \Leftrightarrow \text { instance_of }(v, \text { Death }) \wedge \\
\exists w: \text { instance of }(w, \text { Tissue }) \wedge \text { located_in }(v, w)
\end{gathered}
$$

$\forall x$ : instance_of $(x$, Apoptosis $) \Rightarrow$ instance_of $(x$, Death $) \wedge$ $\exists y$ : instance of $(y$, Cell $) \wedge$ located_in $(x, y)$ 
The above statement would not necessarily hold if every instance of Cell were part_of, and consequently located_in, some instance of Tissue. This is not the case because there are cells which are not part of a tissue. However we could modify the example as follows:

\section{Example 3}

"Neuron Apoptosis is a kind of cell death. Neural tissue has cells as parts. Even though, neuron apoptosis is not a necrosis of neural tissue".

Using the same inference as above we would, indeed, not be able to explain why apoptosis is not to be subsumed by necrosis. A closer look at Formula 14 reveals that "any death process located in some tissue" is a necrosis. Following this definition, of course, any isolated apoptosis event in a tissue would be equivalent to necrosis. This is certainly not what the term "necrosis" means. We will, therefore, have to correct the above definition, using the relation participant_of as introduced in Section 3.3:

$$
\begin{gathered}
\forall v \text { : instance_of }(v, \text { Necrosis }) \Leftrightarrow \text { instance_of }(v, \text { Death }) \wedge \\
\exists w: \text { instance of }(w, \text { Tissue }) \wedge \text { participant_of }(w, v)
\end{gathered}
$$

For the proper definition of biological occurrents, as a general rule, we have to carefully distinguish between a biological object which is the location of an occurrent (i.e. the occurrent may occur even in a small part of this object), or biological objects which are participants of this occurrent, e.g. they are involved in the occurrent as wholes.

We underline this distinction by the following quite trivial medical example:

\section{Example 4}

"Amputation of a foot is an amputation which targets a foot."

$$
\forall x \text { : instance_of }(x, \text { Amputation } \text { Of Foot }) \Leftrightarrow
$$$$
\text { instance_of }(x, \text { Amputation }) \wedge \exists y \text { : instance of }(y, \text { Foot }) \wedge
$$

$$
\text { participant_of }(y, x)
$$

"Amputation at a foot is an amputation which is located at a foot."

$$
\begin{gathered}
\forall x: \text { instance_of }(x, \text { AmputationAtFoot }) \Leftrightarrow \\
\text { instance_of }(x, \text { Amputation }) \wedge \\
\exists y: \text { instance of }(y, \text { Foot }) \wedge \text { located_in }(x, y)
\end{gathered}
$$

As a consequence, any instance AmputationOfToe would be an AmputationAtFoot because every AmputationOfToe has some Toe as participant and every Toe is located_in some Foot, given the transitivity of the relation located_in. Note the subtle but important semantic distinction between the prepositions "at" or "in" (location) and "of" (participant).

The already discussed distinction in propagation patterns between Nephritis and Gastroenteritis can be explained by the same terms. Whilst Nephritis is an inflammatory disorder "at", Gastroenteritis is not only an inflammatory disorder "at" but also an inflammatory disorder "of". Therefore, Glomerulonephritis Is-A Nephritis, whereas Gastroenteritis does not subsume Appendicitis. This way, domain-specific specialized relations such as inflammation-of, fracture-of, necrosis-of, amputation-of (such as proposed in [42]) can easily be reduced to a small set of foundational relations, such as participant_of and located_in, an argument perfectly in line with the requirement of parsimony formulated in [44].

Finally, the propagation patterns fundamentally depends on the distinction between the direction of the class-level partonomic relation, such as illustrated in Fig. 6 and discussed in Section 4.1. This explains the reason why Mitosis is not a Liver process although every Liver has instances of Cell as parts. The explanation is that whereas for each instance of Liver there are instances of Cell and Mitosis, not every Cell is part of a Liver, and therefore not every instance of Mitosis is located at some instance of Liver.

\subsection{An Attempt to Generalize}

The two reasoning patterns, which had been accounted for in the SEP approach (cf. Fig. 5) by relating to the Snodes (for enabling propagation) or to the E-nodes (for obviating propagation) can be reconstructed as follows.

First of all, we reinterpret the additional nodes in a straightforward way. The P-nodes for parts are axiomatized as reificators of the relation proper_part_of:

$$
\begin{gathered}
\forall p: \text { instance_of }\left(p, X_{P}\right) \Leftrightarrow(\exists x: \text { instance_of }(x, X) \wedge \\
\text { proper_part_of }(p, x))
\end{gathered}
$$

while the S nodes as reificators of the relation part_of, formalized as follows:

$$
\begin{gathered}
\forall s: \text { instance_of }\left(s, X_{S}\right) \Leftrightarrow(\exists x: \text { instance_of }(x, X) \wedge \\
\text { part_of }(s, x))
\end{gathered}
$$

The subsumption of P-nodes by S-nodes can be deduced from the fact that proper_part_of implies part_of.

As the relation part_of implies located_in, we obtain the following implication for P-nodes:

$$
\begin{gathered}
\forall p: \text { instance_of }\left(p, X_{P}\right) \Rightarrow(\exists x: \text { instance_of }(x, X) \wedge \\
\text { located_in }(p, x))
\end{gathered}
$$


and for the $S$ nodes accordingly:

$$
\begin{gathered}
\forall x: \text { instance_of }\left(s, X_{S}\right) \Rightarrow(\exists x: \text { instance_of }(x, X) \wedge \\
\text { located_in }(s, x))
\end{gathered}
$$

Consequently, by using the same relation located_in to relate an occurrent with a continuant (instead of using the ill-defined relation associated_with), we obtain the conclusion that an occurrent located at anything subsumed by $X_{S}, v i z$. an occurrent located at any part of some $X$ is located at some $X$ due to the transitivity property of located_in.

Thus, the propagation of properties is a natural consequence of generalizing the notion of "location". If a process $p$ is located_in $x$ and $x$ is located_in $y$ (which is the consequence of $x$ being part_of $y$ ), then the process $p$ is located_in $y$, as well. Coming back to the example in Fig. 5, every instance of Glomerulonephritis is located_in some Glomerula, all Glomerula are part of some Kidney and therefore they are located_in some Kidney. Due to the definition of Nephritis as a disorder located_in some Kidney, we get the terminological inference that every Glomerulonephritis is a Nephritis.

How can we deal now with the "anomalous" cases? A closer look at these cases reveals that they always target some object as a whole. For instance, Nephrectomy is the removal of the whole kidney, while any partial removal of kidney tissue, e.g. in a biopsy, must not be classified as Nephrectomy.

Now the ОВО relation participant_of comes into the play. As shown in Fig. 5, the node denoting a disease class is linked by this relation to the node denoting the class of biological objects. Again, we have to substitute the fuzzy relation associated_with relation by a canonical one. Since participant_of is not a subrelation of located_in, there is no inference pattern as with the Glomerulonephritis case.

One might still have reservations against the use of the relation participant_of, basically due to the lack of a clear definition. In this case, a solution would be to introduce the relation exactly_located_in, according to [10], which is defined as the exact coincidence of two regions.

$$
\begin{gathered}
\forall x, y: \text { exactly_located_in }(x, y)={ }_{\text {def }}(\text { region_of }(x) \\
=\text { region_of }(y))
\end{gathered}
$$

Thus, we continue arguing on the level of spatial relations, without any hidden assumptions which may occur when using an ill-defined relation such as participant_of. Since exactly_located_in is a stricter relation than located_in, the role propagation is obviated. The effect is the same, and the use of exactly_located_in might indeed be preferred due to its semantic clarity. On the other hand, participant_of has been proposed as a foundational relation for bio-ontologies, so that there are good reasons to choose this one. However, this relation is still fuzzy and a substantial clarification of its semantics is due.

\section{Conclusion}

This paper deals with two major issues. The first one is concerned with ontological considerations underlying the substitutability of part-whole by locational relations, in accordance with the OBO (Open Biological Ontologies) Relation Ontology. For physical domains at least, it seems evident to get rid of the ongoing debate about different forms of part-whole reasoning and its underpinnings by abstracting away from part_of/has_part and describing the composition of physical objects in terms of locational relations, viz. located_in and its inverse location_of.

The second issue has to do with typical inferences drawn in the biomedical domain, viz. the propagation of attributes across partonomies. We here propose a straightforward solution where the relation pair located_in/ location_of is used not only to express the spatial relationships between biological objects and spaces but also between occurrents and biological objects. Further work should extend this framework to include biological functions, qualities and dispositions $[20,40]$, as well.

Thus we do refrain from additional formal language constructs in order to obtain the desired inferences (propagation of properties across compositional hierarchies) which are needed in such a reasoning framework. This way, we combine ontological clarity with formal simplicity and descriptional parsimony. We have used first order logics for our formalisms but it can easily be demonstrated that our use of FOL expressiveness translates into OWL-DL [25], the description logics [5] standard of the Semantic Web. The chosen approach is currently used for a principled redesign of the anatomy part of SNOMED CT [3]. There is preliminary evidence that newly designed terminological reasoners [6] are able to efficiently deal with such structures, so that the previous SEP triplet approach - introduced mainly for the purpose to enable efficient computation - can be abandoned.

\section{Authors' contributions}

The method described was principally developed by SS. $\mathrm{KM}$ and $\mathrm{UH}$ participated in the formalization. All authors read and approved the final manuscript.

\section{Acknowledgements}

This work was supported by the EU Network of Excellence "Semantic Interoperability and Data Mining in Biomedicine" (NoE 507505) and the EC STREP project "BOOTStrep" (FP6 - 028099). We also want to thank Martin Romacker (Basel, Switzerland) and Holger Stenzhorn (Saarbrücken, 
Germany) for useful suggestions, as well as Franz Baader and Boontawee Suntisrivaraporn (Dresden, Germany) for fruitful discussions. Finally, we would like to thank the anonymous reviewers for their excellent comments and suggestions that helped to substantially improve the manuscript.

\section{References}

I. Basic Formal Ontology (BFO) 2004 [http://www.ifomis.uni-saarland.de/ bfo/home.php]. Last accessed March 10th, 2007

2. OpenGALEN. The OpenGALEN Foundation 2006 [http:// www.opengalen.org/]. Last accessed March I0th, 2007

3. SNOMED Clinical Terms. Northfield, IL: College of American Pathologists; 2006

4. Artale Alessandro, Franconi Enrico, Guarino Nicola, Pazzi Luca: Partwhole relations in object-centered systems: An overview. Data \& Knowledge Engineering 1996, 20(3):347-383.

5. Baader Franz, Calvanese Diego, McGuinness Deborah L, Nardi Daniele, Patel-Schneider Peter F, editors: The Description Logic Handbook. Theory, Implementation, and Applications Cambridge, U.K.: Cambridge University Press; 2003.

6. Baader Franz, Lutz Carsten, Suntisrivaraporn Boontawee: CEL - a polynomial-time reasoner for life science ontologies. Proceedings of the 3rd International Joint Conference on Automated Reasoning (IJCAR'06), Seattle, WA, USA, August I7-20, 2006 2006, 287-29 I:.

7. Bittner Thomas: Axioms for parthood and containment relations in bio-ontologies. KR-MED 2004 - Proceedings of the Ist International Workshop on Formal Biomedical Knowledge Representation, Collocated with the 9th International Conference on the Principles of Knowledge Representation and Reasoning (KR 2004) 2004:4-II [http://CEURWS.org/Vol-102/]. Whistler, B.C., Canada, June I, 2004. Bethesda, MD: American Medical Informatics Association (AMIA)

8. Bittner Thomas, Donnelly Maureen: A theory of granular parthood based on qualitative cardinality and size measures. Proceedings of the 4th International Conference on Formal Ontology in Information Systems, number 150 in Frontiers in Artificial Intelligence and Applications 2006:65-76.

9. Bittner Thomas, Maureen Donnelly: The mereology of stages and persistent entities. In ECAI 2004 - Proceedings of the 16th European Conference on Artificial Intelligence Edited by: Ramon López de Mántaras, Lorenza Saitta. Valencia, Spain, August 22-27, 2004. Amsterdam: IOS Press; 2004:283-287.

10. Casati Roberto, Varzi Achille C: Parts and Places. The Structures of Spatial Representation Cambridge, MA: MIT Press/Bradford; 1999.

II. Donnelly Maureen, Bittner Thomas, Rosse Cornelius: A formal theory for spatial representation and reasoning in biomedical ontologies. Artificial Intelligence in Medicine 2006, 36(I): I-27.

12. European Bioinformatics Institute. Chemical entities of biological interest (ChEBI) 2006.

13. Fillmore Charles J: The case for case. In Universals in Linguistic Theory Edited by: Emmon Bach, Robert Harms. New York: Holt, Rinehart and Winston; 1968:I-88.

14. Gene Ontology Consortium: Creating the Gene Ontology resource: Design and implementation. Genome Research 200I, I I(8): | 425-| 433.

15. Gene Ontology Consortium. GO editorial style guide 2006 [http://www.geneontology.org/GO.usage.html]. Last accessed: March 10th, 2007

16. Grenon Pierre, Smith Barry: SNAP and SPAN. towards dynamic spatial ontology. Spatial Cognition and Computation 2004, 4:69-I03.

17. Grenon Pierre, Smith Barry, Goldberg Louis: Biodynamic ontology: Applying BFO in the biomedical domain. Proceedings of the Workshop on Medical Ontologies, Rome, October 2003 2004:20-38.

18. Hartel Frank W, de Coronado Sherri, Dionne Robert, Fragoso Gilberto, Golbeck Jennifer: Modeling a description logic vocabulary for cancer research. Journal of Biomedical Informatics 2005, 38(2): I I4-I29.

19. Horrocks lan, Rector Alan L, Goble Carole A: A description logic based schema for the classification of medical data. KRDB'96 - Proceedings of the 3rd Workshop 'Knowledge Representation Meets Databases' :24-28 [http://CEUR-WS.org/Vol-4/]. Budapest, Hungary August 13, 1996

20. Jansen Ludger: On ascribing dispositions. Dispositions and causal powers $2006 \quad$ [http://home.arcor.de/metaphysicus/Texte/ paris artikel6.pdf]. Aldershot: Ashgate

21. Johansson Ingvar, Smith Barry, Munn Katherine, Tsikolia Nikoloz, Elsner Kathleen, Ernst Dominikus, Siebert Dirk: Functional anat- omy: A taxonomic proposal. Acta Biotheoretica 2005, I4(3): I53-166.

22. Lenat Douglas B, Guha Ramanathan V: Building Large Knowledge-Based Systems. Representation and Inference in the Cyc Project Reading, MA: Addison-Wesley; 1990

23. ОВO: Open Biological Ontologies (OBO) 2005 [http://obo.source forge.net]. Last accessed March I0th, 2007

24. Patel-Schneider Peter F: What's inheritance got to do with knowledge representation? In Inheritance Hierarchies in Knowledge Representation and Programming Languages Edited by: Maurizio Lenzerini, Daniele Nardi, Maria Simi. Chichester: John Wiley; I99।:I-I I.

25. Patel-Schneider Peter F, Hayes Pat, Horrocks lan: OWL Web Ontology Language; Semantics and Abstract Syntax. 2004.

26. Rector Alan, Rogers Jeremy, Bittner Thomas: Granularity scale and collectivity: When size does and doesn't matter. Journal of Biomedical Informatics 2005, 38:333-349.

27. Rector Alan L: Analysis of propagation along transitive roles: Formalisation of the GALEN experience with medical ontologies. DL 2002 - Proceedings of the 2002 International Workshop on Description Logics [http://CEUR-WS.org/Vol-53/]. Toulouse, France April 19-2I, 2002

28. Rector Alan L, Bechhofer Sean, Goble Carole A, Horrocks lan, W Anthony Nowlan, W Danny Solomon: The GRAIL concept modelling language for medical terminology. Artificial Intelligence in Medicine 1997, 9(2): |39-17|.

29. Rector Alan L, Gangemi Aldo, Galeazzi Elena, Glowinski Andrzej J, Mori Angelo Rossi: The GALEN model schemata for anatomy: Towards a re-usable application-independent model of medical concepts. In MIE'94 - Medical Informatics Europe 94. Proceedings of the I2th Conference of the European Federation for Medical Informatics Edited by: Barahona P, Veloso M, Bryant J. Lisbon, Portugal, 22-26 May 1994. Amsterdam: IOS Press; 1994:229-233.

30. Rogers Jeremy, Rector Alan L: GALEN's model of parts and wholes: Experience and comparisons. In AMIA 2000 - Proceedings of the Annual Symposium of the American Medical Informatics Association. Converging Information, Technology, and Health Care Edited by: Marc Overhage J. Los Angeles, CA, November 4-8, 2000. Philadelphia, PA: Hanley \& Belfus; 2000:714-7I8.

31. Rosse Cornelius, Mejino José Leonardo V: A reference ontology for bioinformatics: the Foundational Model of Anatomy. Journal of Biomedical Informatics 2003, 36:478-500.

32. Rosse Cornelius, Mejino José Leonardo V, Modayur Bharath R, Jakobovits Rex, Hinshaw Kevin P, Brinkley James F: Motivation and organizational principles for anatomical knowledge representation: The DIGITAL ANATOMIST symbolic knowledge base. Journal of the American Medical Informatics Association 1998, 5(I): $17-40$.

33. Samwald Matthias: Classes versus individuals: Fundamental design issues for ontologies on the biomedical semantic web. In Integrating Biomedical Information: from eCell to ePatient Edited by: Reichert A, Mihalas G, Stoicu-Tivadar L, Schulz S, Engelbrecht R. Amsterdam: IOS Press; 2006:335-340.

34. RO: OBO Relation Ontology 2007 [http://obo.sourceforge.net/relation ship/relationship.obo-xml].

35. Schulz Stefan, Daumke Philipp, Smith Barry, Hahn Udo: How to distinguish parthood from location in bio-ontologies. In AMIA 2005 - Proceedings of the Annual Symposium of the American Medical Informatics Association. Biomedical Informatics: From Foundations to Applications to Policy Edited by: Charles P Friedman. ; 2005:669-673.

36. Schulz Stefan, Hahn Udo: Mereotopological reasoning about parts and (w)holes in bio-ontologies. In Formal Ontology in Information Systems. Collected Papers from the 2nd International FOIS Conference Edited by: Chris Welty, Barry Smith. Ogunquit, Maine, USA, October 17-19, 200I. New York, NY: ACM Press; 2001:210-22I.

37. Schulz Stefan, Hahn Udo: Necessary parts and wholes in bioontologies. In Principles of Knowledge Representation and Reasoning. Proceedings of the 8th International Conference - KR 2002 Edited by: Fensel D, Giunchiglia F, McGuinness D, Williams M-A. Toulouse, France, April 22-25, 2002. San Francisco, CA: Morgan Kaufmann; 2002:387-394.

38. Schulz Stefan, Hahn Udo: Part-whole representation and reasoning in biomedical ontologies. Artificial Intelligence in Medicine 2005, 34(3): 179-200.

39. Schulz Stefan, Hanser Susanne, Hahn Udo, Rogers Jeremy: The semantics of procedures and diseases in snomed ct. Methods of Information in Medicine 2006, 45(4):354-358. 
40. Schulz Stefan, Jansen Ludger: "Imo-2 interacts with elf-2". on the meaning of common statements in biomedical literature. KR-MED 2006 - Proceedings of the 2nd International Workshop on Formal Biomedical Knowledge Representation 2006:37-45 [http://CEURWS.org/Vol-222/]. Baltimore MD, November 8, 2006

41. Schulz Stefan, Kumar Anand: Biomedical ontologies: What partof is and isn't. Journal of Biomedical Informatics 2006, 39(3):350-36I.

42. Schulz Stefan, Romacker Martin, Hahn Udo: Part-whole reasoning in medical ontologies revisited: Introducing SEP triplets into classification-based description logics. In AMIA'98 - Proceedings of the 1998 AMIA Annual Fall Symposium. A Paradigm Shift in Health Care Information Systems: Clinical Infrastructures for the 21st Century Edited by: Chute CG. Orlando, FL, November 7-II, 1998. Philadelphia, PA: Hanley \& Belfus,; 1998:830-834.

43. Simons Peter: Parts: A Study in Ontology Oxford: Clarendon Press; 1987.

44. Smith Barry, Ceusters Werner, Klagges Bert, Köohler Jacob, Kumar Anand, Lomax Jane, Mungall Chris, Neuhaus Fabian, Rector Alan L, Rosse Cornelius: Relations in biomedical ontologies. Genome Biology 2005, 6(5):R46.

45. Smith Barry, Rosse Cornelius: The role of foundational relations in the alignment of biomedical ontologies. In MEDINFO 2004 - Proceedings of the IIth World Congress on Medical Informatics. vol I, number 107 in Studies in Health Technology and Informatics Edited by: Marius Fieschi, Enrico Coiera, Yu-Chan Jack Li. San Francisco, CA, USA, September 7-I I, 2004. Amsterdam: IOS Press; 2004:444-448.

46. Varzi Achille C: Mereology. In Stanford Encyclopedia of Philosophy Edited by: Edward N Zalta. Stanford: The Metaphysics Research Lab; 2003.

47. Varzi Achille C: A note on the transitivity of parthood. Applied Ontology 2006: | $4|-| 46$.

\section{Publish with Bio Med Central and every scientist can read your work free of charge}

"BioMed Central will be the most significant development for disseminating the results of biomedical research in our lifetime. "

Sir Paul Nurse, Cancer Research UK

Your research papers will be:

- available free of charge to the entire biomedical community

- peer reviewed and published immediately upon acceptance

- cited in PubMed and archived on PubMed Central

- yours - you keep the copyright

Submit your manuscript here:

http://www.biomedcentral.com/info/publishing_adv.asp
Biomedcentral 